\title{
Modeling Spatial Effects in Early Carcinogenesis: Stochastic Versus Deterministic Reaction-Diffusion Systems
}

\author{
R. Bertolusso ${ }^{1}$ and M. Kimmel ${ }^{1,2 *}$ \\ ${ }^{1}$ Department of Statistics, Rice University, 6100 Main Street, MS138, Houston, TX 77005, USA \\ ${ }^{2}$ Systems Engineering Group, Silesian University of Technology, 44-100 Gliwice, Poland
}

\begin{abstract}
We consider the early carcinogenesis model originally proposed as a deterministic reaction-diffusion system. The model has been conceived to explore the spatial effects stemming from growth regulation of pre-cancerous cells by diffusing growth factor molecules. The model exhibited Turing instability producing transient spatial spikes in cell density, which might be considered a model counterpart of emerging foci of malignant cells. However, the process of diffusion of growth factor molecules is by its nature a stochastic random walk. An interesting question emerges to what extent the dynamics of the deterministic diffusion model approximates the stochastic process generated by the model. We address this question using simulations with a new software tool called sbioPN (spatial biological Petri Nets). The conclusion is that whereas singlerealization dynamics of the stochastic process is very different from the behavior of the reaction diffusion system, it is becoming more similar when averaged over a large number of realizations. The degree of similarity depends on model parameters. Interestingly, despite the differences, typical realizations of the stochastic process include spikes of cell density, which however are spread more uniformly and are less dependent of initial conditions than those produced by the reactiondiffusion system.
\end{abstract}

Key words: cancer modelling, deterministic, stochastic, reaction-diffusion equations, pattern formation, spike solutions

AMS subject classification: $35 \mathrm{~K} 57,92 \mathrm{C} 15$

\footnotetext{
${ }^{*}$ Corresponding author. E-mail: kimmel@rice.edu
} 


\section{Deterministic reaction-diffusion model of early carcinogen- esis}

\subsection{Hypotheses and equations}

The model of a pre-cancerous cell population is as in reference [1], which adopts elements of the models previously proposed in references $[2,3]$. The present model is based on the following hypotheses:

- Pre-cancerous cells $c$, existing in a spatial domain, proliferate at a rate $a(b, c)$, which is reduced by cell crowding but enhanced in a paracrine manner by a hypothetical bio-molecular growth factor $b$ bound to cells.

- Pre-cancerous cells are supplied at a constant rate $\mu$ by mutation of normal cells.

- Free growth factor $g$ is secreted by the cells at the rate $\kappa(c)$, then it diffuses among cells with diffusion constant $1 / \gamma$, and binds to cell membrane receptors at a rate $\alpha(c)$, becoming the bound factor $b$. It then dissociates at a rate $d$, returning to the free factor pool.

- Free and bound growth factor particles decay at rates $d_{g}$ and $d_{b}$, respectively.

Discussion of possible geometries for the spatial variable $x$ can be found in previous papers [2, 4]. One natural geometry is that of a line of cells, occupying the interval $x \in[0,1]$. There are three substances distributed over the line's length: Cells and free and bound growth factor molecules, with densities $c(x, t), g(x, t)$ and $b(x, t)$, respectively. The resulting equations are as follows:

$$
\begin{aligned}
\frac{\partial c}{\partial t} & =\left(a(b, c)-d_{c}\right) c+\mu, \\
\frac{\partial b}{\partial t} & =\alpha(c) g-d_{b} b-d b, \\
\frac{\partial g}{\partial t} & =\frac{1}{\gamma} \frac{\partial^{2} g}{\partial x^{2}}-\alpha(c) g-d_{g} g+\kappa(c)+d b,
\end{aligned}
$$

with homogeneous Neumann (zero flux) boundary conditions for $g$

$$
\partial_{x} g(0, t)=\partial_{x} g(1, t)=0
$$

The kinetics were derived from the stochastic model describing the transitions between different states of the growth factor molecules [2,4]. Diffusion equation is a macroscopic approximation of the microscopic process of growth factor binding to membrane receptors, under homogeneity hypotheses. A deterministic derivation based on homogenization methodology has been published in reference [5]. Coefficient $1 / \gamma$ is a composite parameter including the diffusion constant and scaling parameters, $\gamma=1 / D$. Proliferation rate has the Hill function form

$$
a(c, b)=\frac{a_{1}(b / c)^{m}}{1+(b / c)^{m}}
$$


where $a_{1}=(2 p-1) a_{0}$ and $p$ is the efficiency of divisions. We will consider the special case $m=1$. Production of free growth factor by cells has the Michaelis-Menten form

$$
\kappa(c)=\frac{\kappa c}{1+c}
$$

The form of growth factor binding rate $\alpha(c)$ has been obtained from conditions for diffusion-driven instability investigated in ref. [4]. It assumes the form,

$$
\alpha(c)=\alpha_{1} c^{s+1} \quad s>0
$$

i.e., the process of binding free growth factor particles to cells is super-linear.

\subsection{Spatially homogeneous steady state}

¿From reference [1], the spatially homogeneous steady states of pre-cancerous cells $(\bar{c})$ are the solutions to:

$$
f_{1}(c) \equiv \kappa \frac{c^{2}}{d_{g} \frac{d_{b}+d}{\alpha}(1+c)+\kappa c^{2}+d_{b} c^{2}(1+c)}=-\frac{\mu}{a_{1} c}+\frac{d_{c}}{a_{1}} \equiv f_{2}(c)
$$

The corresponding expression for the bound growth factor is:

$$
\bar{b}=\kappa \frac{\bar{c}}{1+\bar{c}} \frac{\bar{c}^{2}}{d_{b} \bar{c}^{2}+d_{g} \frac{d_{b}+d}{\alpha}}
$$

and for the free growth factor:

$$
\bar{g}=\frac{d_{b}+d}{\alpha \bar{c}^{2}} \bar{b}
$$

For the parameter values adopted for this study and summarized in Table 1, the solution of $\bar{c}$ is unique (case (2) in Proposition 1).

\begin{tabular}{|c||c|}
\hline Parameter & Value \\
\hline$a_{1}$ & $1 / 12$ \\
$\alpha$ & $10^{-1}$ \\
$\kappa$ & 1 \\
$s$ & 1 \\
$d_{c}$ & $5 \times 10^{-2}$ \\
$\mu$ & $10^{-2}$ \\
$d=d_{b}=d_{g}$ & $10^{-1}$ \\
\hline
\end{tabular}

Table 1: Values of the parameters used in all studied systems.

The resulting equilibrium values are $\bar{c}=5.86, \bar{b}=8.07$, and $\bar{g}=0.47$. Numerical integration of the deterministic system agrees with these results, which are presented in the left column of Figure 2. This is the same case as in the right panel of Figure 1 and in Figure 7, in Reference [1]. 
Existence of inhomogeneous steady states has been discussed in Reference [1]. Briefly, such solutions have to satisfy a second order ODE (see further on) with appropriate boundary conditions.

The results in Reference [1] can be summarized by the following

Proposition 1. Under some assumptions on the model parameters (see Theorems 3.6 and $3.10 \mathrm{in}$ Reference [1] for detail) the system analyzed in the present paper and in Reference [1] has the following stationary solutions:

- Spatially homogeneous steady states defined as equilibria of the kinetics system. As explained in Section 3.2 of Reference [1] two special cases can be distinguished, depending on whether the ratio $d_{c} / a_{1}$ is greater or smaller compared to the maximum of function $f_{1}$, defined in Expression (1.8). We limit ourselves to the former possibility (for further details see Reference [1]).

1. If the maximum of function $f_{1}$ is greater than $d_{c} / a_{1}$, then there exist three nonnegative roots for $\mu$ below a threshold. The exact pattern of stability of these equilibria seems to be difficult to investigate analytically because of the complicated form of algebraic conditions for stability. Numerically, it appears that the smallest nonnegative steady state is always stable, the second one is always unstable, and the third steady state (the greatest one) can be destabilised by diffusion.

2. When $\mu$ is larger than the threshold only one spatially homogeneous equilibrium exists. The stability of this state depends on the value of $\mu$ (Corollary 3.12 in Reference [1]). There exists another threshold such that for $\mu$ above this second threshold destabilisation condition is not satisfied (c.f. also Lemma 3.4 in Reference [1]).

- Unique strictly increasing solution and a unique strictly decreasing solution.

- Periodic solution $W$ with $n$ modes, increasing in intervals $\left[0, \frac{1}{n}\right]$ and its symmetric counterpart $\tilde{W}(x) \equiv W_{n}(1-x)$, where $n \in \mathbb{N}$ depends on the diffusion coefficient; and the periodic function $W \in C([0,1])$ is defined by the following

$$
W(x)=\left\{\begin{aligned}
W\left(x-\frac{2 j}{n}\right) & ; \quad x \in\left[\frac{2 j}{n}, \frac{2 j+1}{n}\right] \\
W\left(\frac{2 j+2}{n}-x\right) & ; \quad x \in\left[\frac{2 j+1}{n}, \frac{2 j+2}{n}\right]
\end{aligned}\right.
$$

for every $j \in\{0,1,2,3, \cdots\}$ such that $2 j+2 \leq n$.

In Reference [1] such solutions where constructed. Preprint [6] is devoted to the analysis of the special version of the model with $\mu=0$ and a constant $\kappa(c)=\kappa_{0}$. In this case, it was shown that all smooth stationary solutions are of the form given above. The result seems to hold also for positive $\mu$. In Reference [6] it was also shown that with $\mu=0$ and a constant $\kappa(c)=\kappa_{0}$ all stationary solutions are unstable (including also the solutions which are not smooth). The extension of this result to the model with a positive $\mu$ does not seem to be straightforward. Nevertheless, numerical simulations of the deterministic model performed in Reference [1] and also in the present manuscript seem to be qualitatively in agreement with the study of the model with $\mu=0$ and a constant $\kappa(c)=\kappa_{0}$. 


\subsection{Perturbation of the spatially homogeneous steady state}

Cosinusoidal perturbation is applied to $\bar{c}$ resulting in initial condition of the form

$$
c_{0}=\bar{c}+\epsilon \cos (2 n \pi x), \quad 0 \leq x \leq 1
$$

where $\epsilon=10^{-2}$ is the amplitude used [1], and $n$ is the number of peaks. If the spatially homogeneous steady state is unstable, this is sufficient for the numerical solutions to be repelled.

\subsection{Numerical integration of the perturbed system}

Ten different grid densities $(5 ; 10 ; 25 ; 50 ; 75 ; 100 ; 150 ; 200 ; 300 ;$ and 400 nodes $)$, five different $\gamma$ values $(\gamma=1 ; 10 ; 100 ; 1000$; and 10,000), and five different $n$ values ( 1 to 5 ) were considered. As a consequence, we studied a total of two hundred and fifty perturbed deterministic reactiondiffusion systems. In all cases, including the stochastic simulations, other parameter values remained constant (Table 1).

Each perturbed deterministic system was numerically integrated for 2000 time units, and the values at 2000 time units, rounded to the nearest integer, were used as the initial conditions for stochastic simulations.

We call the "main case" the one having the parameter values $\gamma=100$, and $n=5$, as in reference [1]. It shares the same characteristics as other cases with equal $\gamma$, but its behavior is different compared to the cases with other $\gamma$ values. Those cases will be reviewed in Section 3. We will start by only considering a grid density of 100 nodes. We will later (Section 4.) analyze the change of behavior of the main case as the grid density changes.

Time evolution of the main case is presented in the left column of Figure 3. The top portion shows the time evolution of pre-cancerous cells, and the bottom portion the corresponding time evolution of free growth factor. Bound growth factor is not included in the plots as has almost exactly the same shape as the plot of the pre-cancerous cells.

Numerical integration of the main case shows, at 2000 time units, a symmetric configuration of four full spikes and two "half-spikes" at the boundaries. Extending integration to 4000 time units does not lead to a change in behavior.

\section{Stochastic reaction-diffusion model}

Stochastic version of the model has been simulated using the sbioPN software developed in the doctoral thesis of Roberto Bertolusso [7]. In the set-up used, cells and bound growth factor particles are immobile, but free growth factor molecules are mobile. Cell proliferation, binding and unbinding of growth factor particles, as well as diffusion are modeled as stochastic chemical reactions in space represented by a periodic quadratic grid. System trajectories are paths of the free growth factor molecules and counts of the cells and of the bound growth factor molecules. Since it would be difficult to depict such trajectories graphically, the plots depict statistics, specifically 
spatial histograms derived from the trajectories. This way of presenting the results allows direct visual comparison with densities produced by the deterministic PDE reaction-diffusion model.

As stated, diffusion has been considered as a succession of two first-order stochastic chemical reactions each with reaction constant $[8,9]$

$$
j=\frac{1}{\gamma h^{2}}
$$

where $h$ is the distance between two grid nodes.

The model was simulated with an optimized variant of the stochastic simulation algorithm known as constant-time composition-rejection algorithm [10], part of the developed library sbioPN [7].

To study the behavior of the stochastic system, simulations with three different initial conditions were performed:

1. with all the initial quantities set to zero: $c(x, 0)=g(x, 0)=b(x, 0)=0$.

2. with the spatially homogeneous steady states rounded to the nearest integer: $c(x, 0)=[\bar{c}]$; $g(x, 0)=[\bar{g}] ;$ and $b(x, 0)=[\bar{b}]$.

3. with the end values of the deterministic perturbed systems rounded to the nearest integer.

Two hundred and fifty stochastic simulations have been performed with the last type of initial conditions, i.e., the same number as in the deterministic case. Rounding to the nearest integer is an unavoidable requirement for stochastic simulation. Further studies showed that rounding did not play a role in the observed differences between deterministic and stochastic systems.

\subsection{Single runs}

One thousand runs were performed of each type of stochastic simulation. In this section we show sample realizations using two of the initial conditions specified above. We only show the evolution of pre-cancerous cells. Results for the bound growth factor, not exactly identical, are very similar to those for pre-cancerous cells up to a rescaling factor, and results for the free growth factor do not show a discernible structure.

Left panel of Figure 1 depicts a typical run with zero initial conditions. Spikes appear in a mostly uniform way along the $x$ axis. They persist for a variable amount of time, subsequently being replaced by spikes formed at different coordinates.

Stochastic simulations with initial conditions being the spatially homogeneous steady state values of the deterministic system rounded to the nearest integer (not shown) rapidly evolve to a behavior similar to those with zero initial values.

The right panel of Figure 1 depicts a typical stochastic runs with initial conditions set to the values of solutions of the perturbed main case at 2000 time units, rounded to the nearest integer. The initial conditions in this case have four full spikes and two "half-spikes" at the boundaries. Results show that the stochastic system does not preserve all the spikes. Most of them disappear rapidly, and no more than two spikes coexist in some runs. 


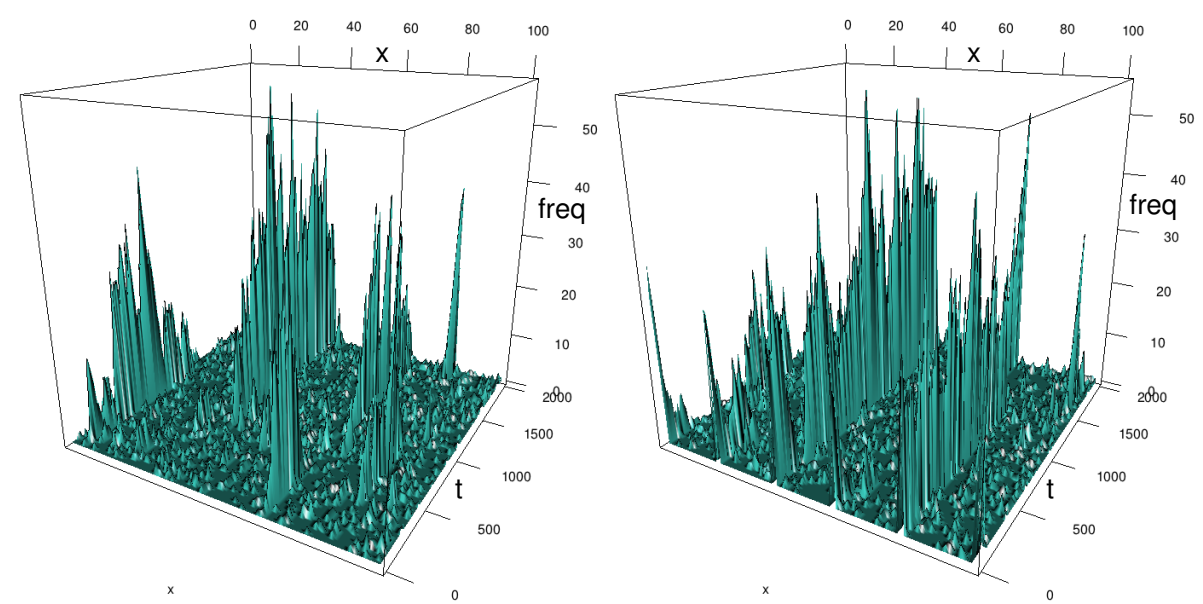

Figure 1: Single runs of the stochastic reaction-diffusion system. Left panel depicts a run with zero initial conditions, while the right panel depicts a run with initial condition set to the end values of the deterministic system rounded to nearest integer. In both cases, $\gamma=100$.

\subsection{Averages of 1000 runs}

Figure 2 shows a comparison between deterministic (unperturbed) and stochastic systems. The average of the number of pre-cancerous cells in the stochastic simulation using zero initial conditions is significantly lower than the result of the deterministic simulation (5.86 compared to values below 1). This behavior is also paralleled by the bound growth factor (not shown). As for the free growth factor, for both types of initial conditions, it seems to converge to values close to the deterministic one.

To further analyze the cause of this behavior, we performed deterministic and stochastic simulations for the associated reaction-only model, and we present the results in the right column of Figure 2. The deterministic and stochastic mean values of the density of pre-cancerous cells differ almost six-fold, in a way similar to what is observed in the spatial case. The deterministic mean values for free growth factor are identical for spatial and non-spatial cases.

However, the stochastic mean of the free growth factor differs with respect to the non-spatial stochastic mean, both in initial shape and in the equilibrium values achieved. The first rapidly increases and decreases at the initial time units, reaching a stable plateau at about 0.4 at time 500 . The second, in contrast, rapidly grows until reaching a plateau at about 0.7 in a very short time.

The initial increase and decrease in mean values for the spatial case may be related to the diffusive characteristic of free growth factor: as it is not produced equally along the $x$ axis, the observed effect may be related to an initial imbalance that is compensated as time progresses. However, this is speculative and it does not explain why the reaction-diffusion and reaction-only systems achieve a different equilibrium level.

Figure 3 shows a comparison of the perturbed deterministic main case system and the averages of 1000 runs of the stochastic system, assuming as initial conditions the values of the deterministic system at time 2000 rounded to the nearest integer. The initial-value spikes of the pre-cancerous cells rapidly fall to about two thirds of the initial value, and then continue to decrease at a lower rate. Based on this alone, it is not clear if after 2000 time units the spike values continue to 

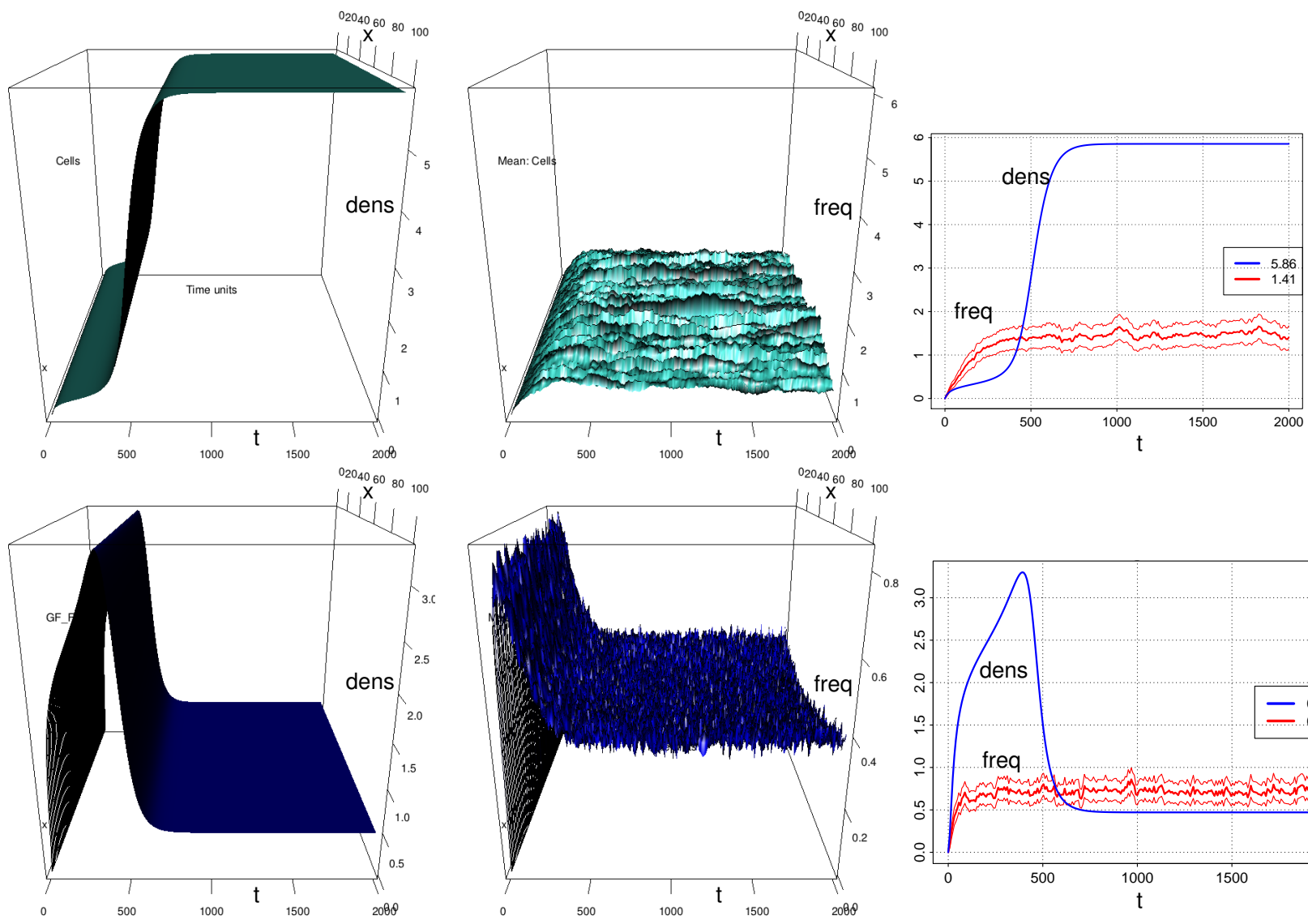

Figure 2: Comparisons of the deterministic and stochastic reaction-diffusion and reaction-only systems. Zero initial conditions are assumed. Depicted are, in the first row, pre-cancerous cells and, in the second row, free growth factor. First column shows deterministic averages, while the second column shows averages of 1000 stochastic runs. Third column shows the reaction-only system (blue lines: deterministic solutions, red lines: averages \pm 3 standard errors of of 1000 stochastic runs). Insets depict values at time 2000. 


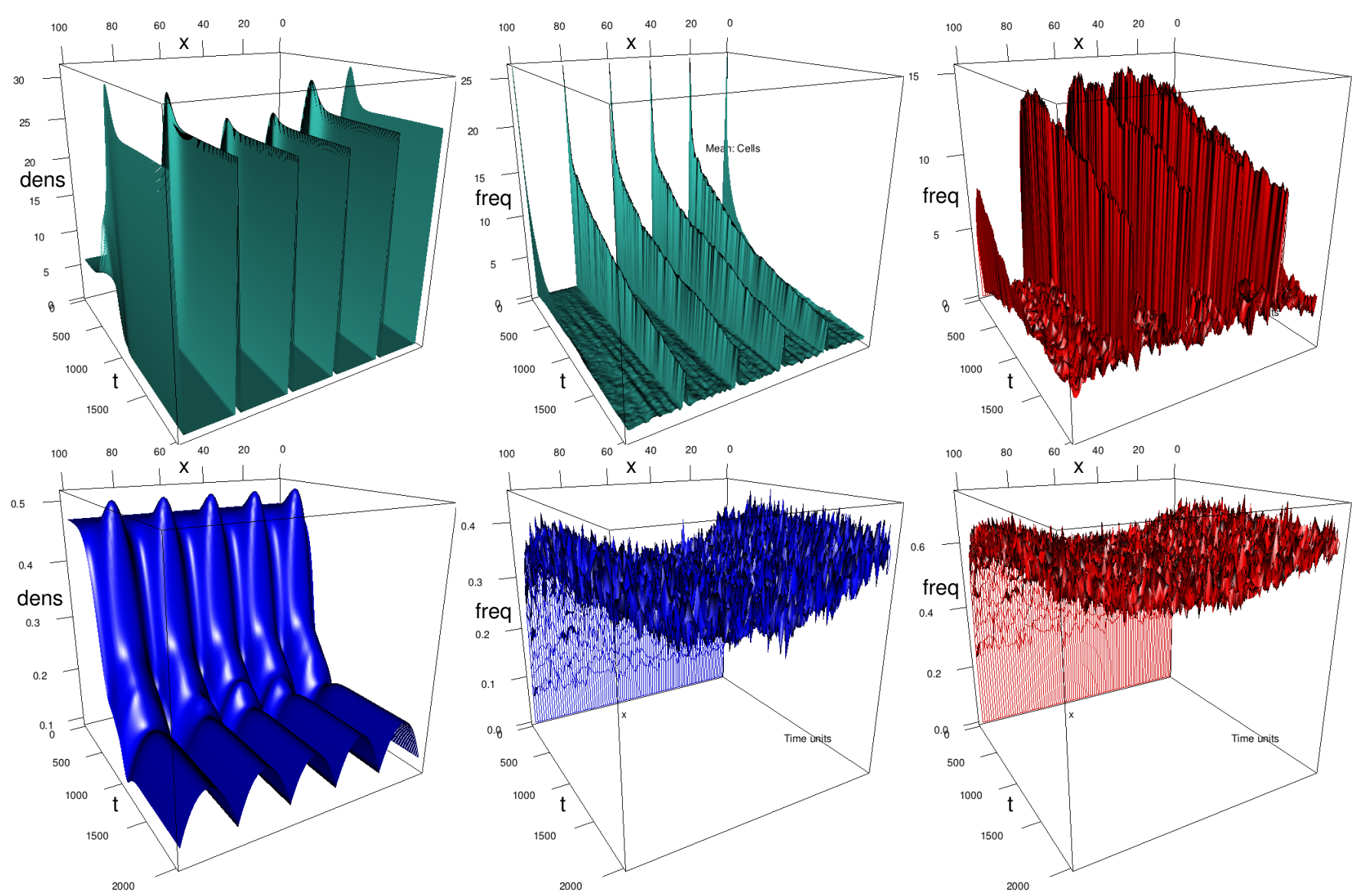

Figure 3: Deterministic versus stochastic reaction-diffusion systems comparison, after perturbation. First row depicts the density of pre-cancerous cells; second row depicts the number of free growth factor molecules. First column: deterministic evolution after perturbation. Second and third columns: averages and standard deviations, respectively, of 1000 stochastic runs with initial conditions set to the values of the deterministic system at time 2000 rounded to the nearest integer. 
decrease until they reach a limit value or until reaching the same level as in Figure 2. Vanishing of the "half-spikes" at the boundaries suggests the second option.

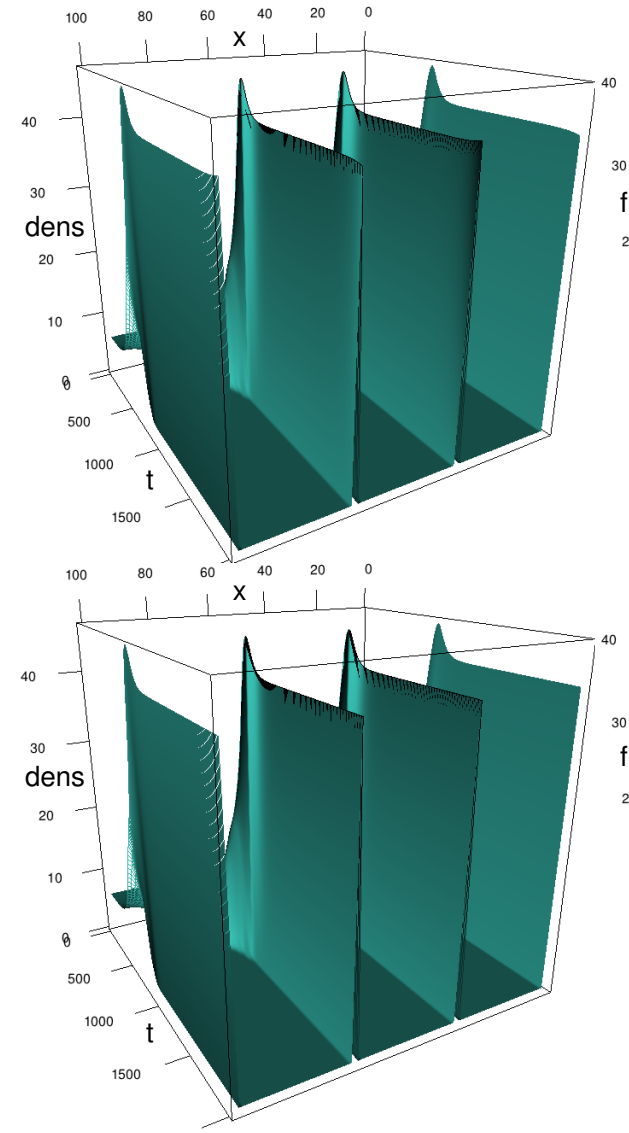

2000
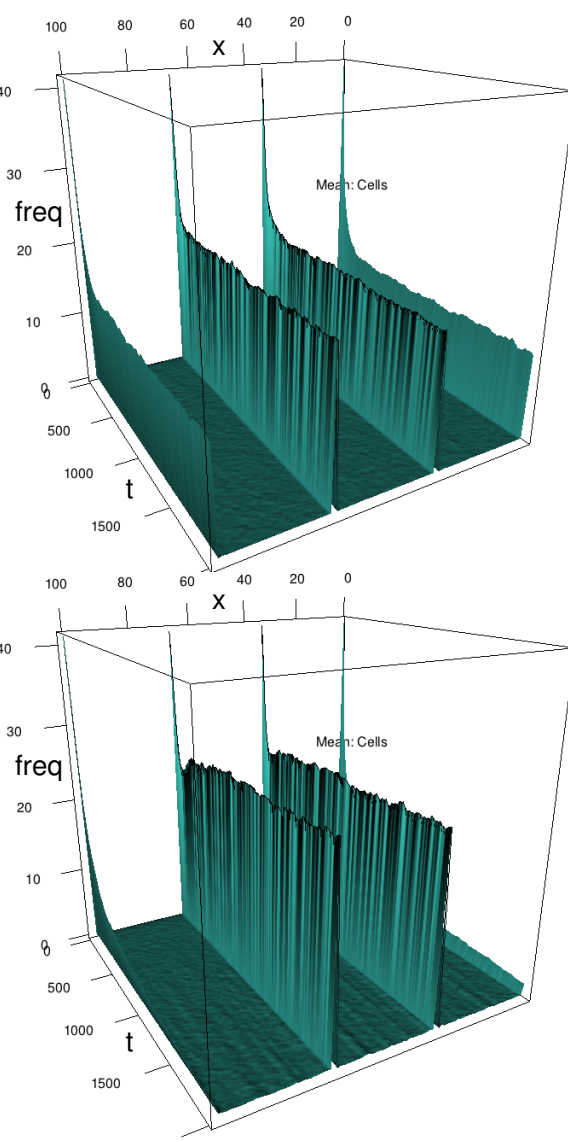

2000
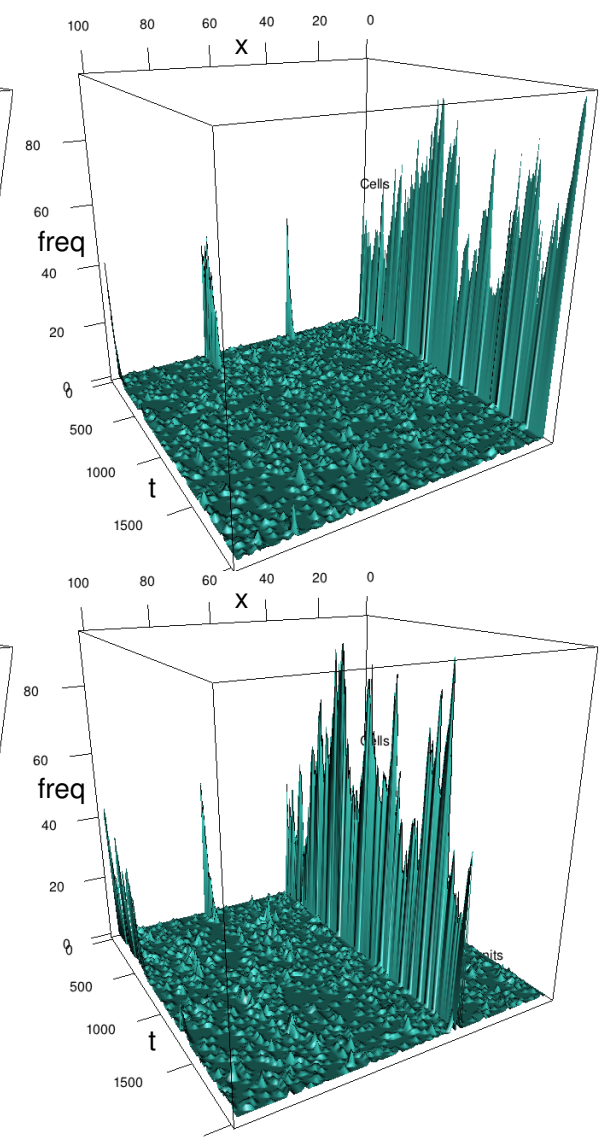

2000

Figure 4: Deterministic versus stochastic system comparison for different values of parameter $\gamma$. First column depicts evolution of perturbed deterministic systems. Second column depicts averages of 1000 stochastic runs. Third column depicts single stochastic runs. The stochastic simulations have initial condition set to the values at time 2000 of the deterministic system rounded to the nearest integer. All cases: $n=3$. First row: $\gamma=1$; second row: $\gamma=10$.

To address this issue a new series of 1000 runs was performed for the main case with time ranging up to 8000 units. The results (not shown) exhibit that, in the long run, the averaged spikes disappear from the stochastic system.

\section{Behavior of the system for different rates of diffusion}

Let us remind that in the so-called main case, the parameter values assumed were $n=5$ and $\gamma=$ 100. In this section we will present a comparison of deterministic and stochastic results obtained when studying the case $n=3$ for the range of $\gamma$ values that we studied: $\gamma=1 ; 10 ; 100 ; 1000$; and 
10,000. As $\gamma$ is the reciprocal of the diffusion constant, lower values of $\gamma$ are equivalent to higher diffusion rates, given equal scaling.
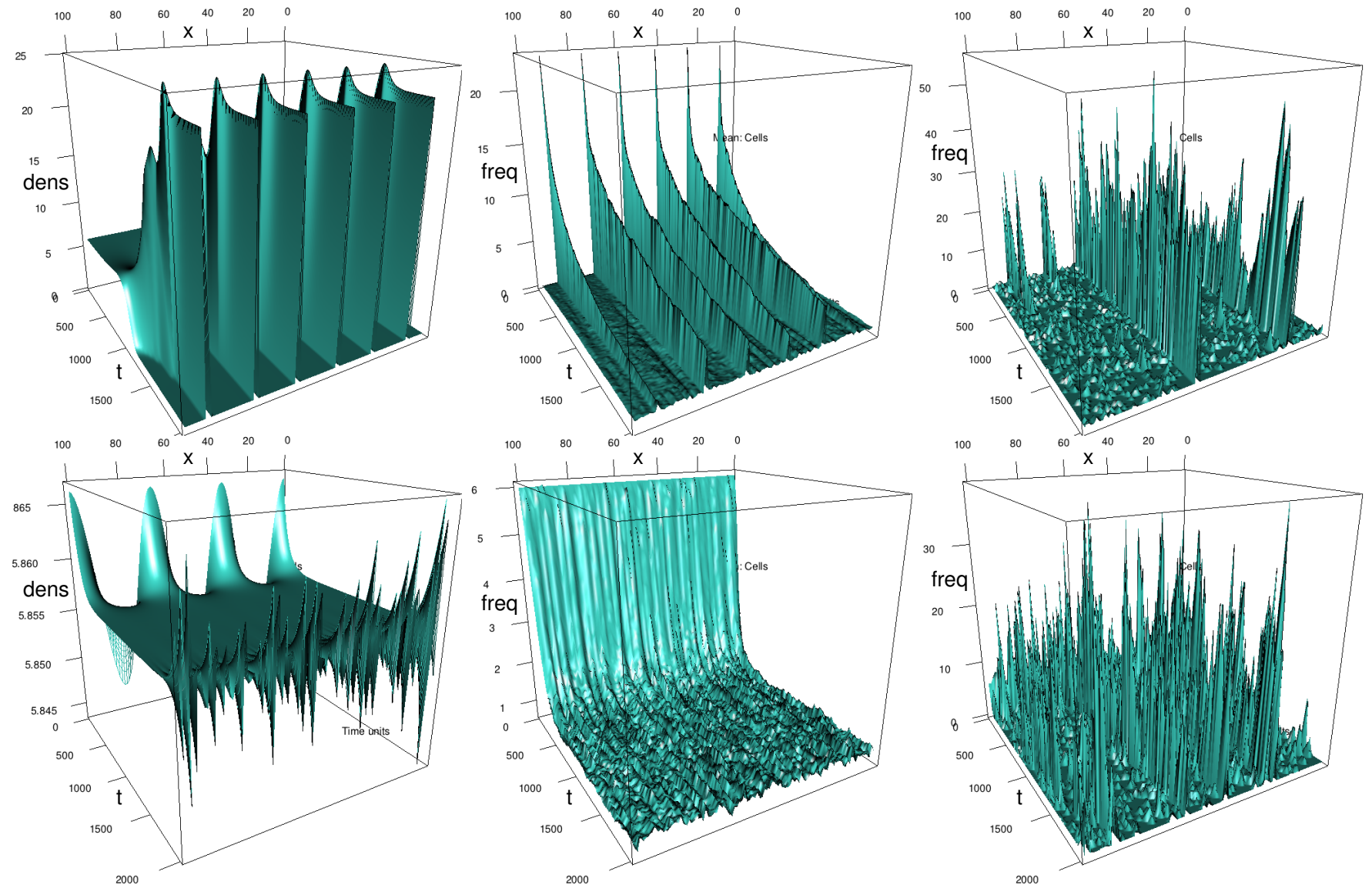

Figure 5: Further deterministic versus stochastic system comparison for different values of parameter $\gamma$. First column depicts evolution of perturbed deterministic systems. Second column depicts averages of 1000 stochastic runs. Third column depicts single stochastic runs. The stochastic simulations have initial condition set to the values at time 2000 of the deterministic system rounded to the nearest integer. All cases: $n=3$. First row: $\gamma=100$; second row: $\gamma=1000$.

We have chosen $n=3$ because the resulting deterministic systems exhibit two full and two "half-spikes" for $\gamma$ assuming values 1 and 10, providing good initial values for the stochastic simulations. Deterministic systems with other $n$ values provide less interesting initial values for these $\gamma$ values (most of them only show the "half-spikes" at the boundaries for at least one of the $\gamma$ values, as it happens in the main case). Other parameter combinations are not shown because they lead to the same conclusions as the case $n=3$.

The results, presented in Figures 4 and 5 for increasing values of $\gamma$, are organized as follows: each figure shows results for two different values of $\gamma$, one in the first row and the other in the second row. Left column shows the deterministic simulations of the perturbed system. Middle column shows the average of 1000 stochastic runs. Right column shows one representative stochastic run. 
Based on the computations, the stochastic system better preserves the averaged spikes of the pre-cancerous cells and the bound growth factor (not shown), if lower values of $\gamma$ are assumed, or, equivalently, if diffusion is higher. It even seems the average spike height is indefinitely preserved in time for $\gamma=1$ (first row of Figure 4). Single runs show that one of the original spikes is maintained through completion of simulation, while the rest disappear rapidly. By observing the plot of the averages, it seems that the internal spikes are preserved with greater probability compared to the boundary spikes.

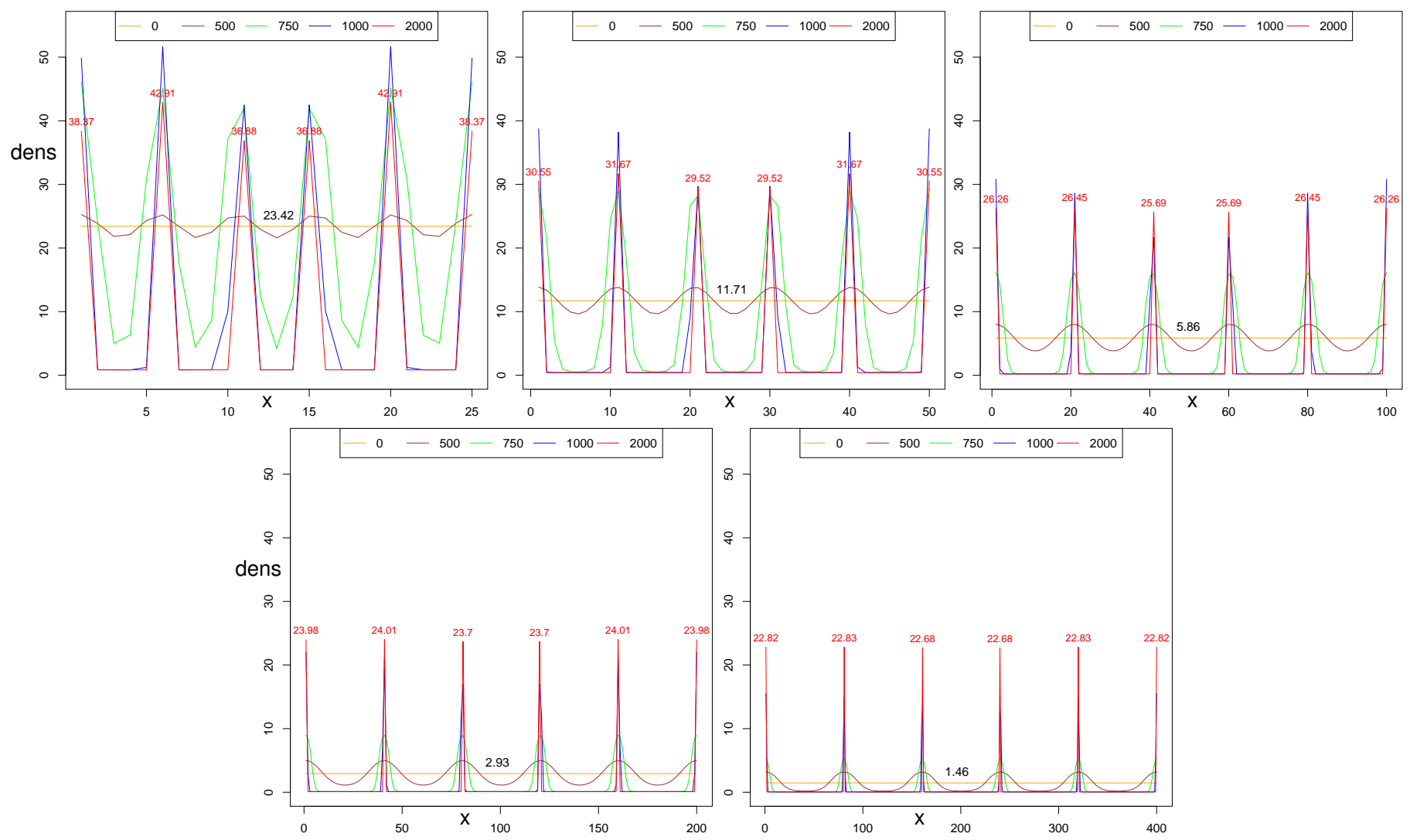

Figure 6: Cross sections of evolution of perturbed deterministic systems for different grid densities. Depicted are densities of the pre-cancerous cells. Horizontal axis shows the number of grid nodes in the interval $0 \leq x \leq 1$.

In the second row of Figure $4(\gamma=10)$ a similar behavior can be observed for the internal but not for the boundary spikes. Single runs show that the boundary spikes disappear rapidly while one of the internal spikes is maintained throughout the duration of simulations.

First row of Figure 5 depicts the case using $\gamma=100$ that, as expected, shows behavior similar to that observed on the main case for the averaged values (Figure 3): a marked drop from the initial values followed by a gradual decay. The same can be said about the similarities found among single runs compared to the ones of the main system (Figure 1).

The bottom portion of Figure 5 depicts how the deterministic system becomes numerically unstable for $\gamma=1000$. In contrast, the stochastic system, exhibits the type of behavior observed in Figure 2. 
Finally, for $\gamma=10,000$ (not shown) both the deterministic and stochastic systems revert to the space-homogeneous non trivial equilibrium state.

\section{Consequences of varying the grid density}

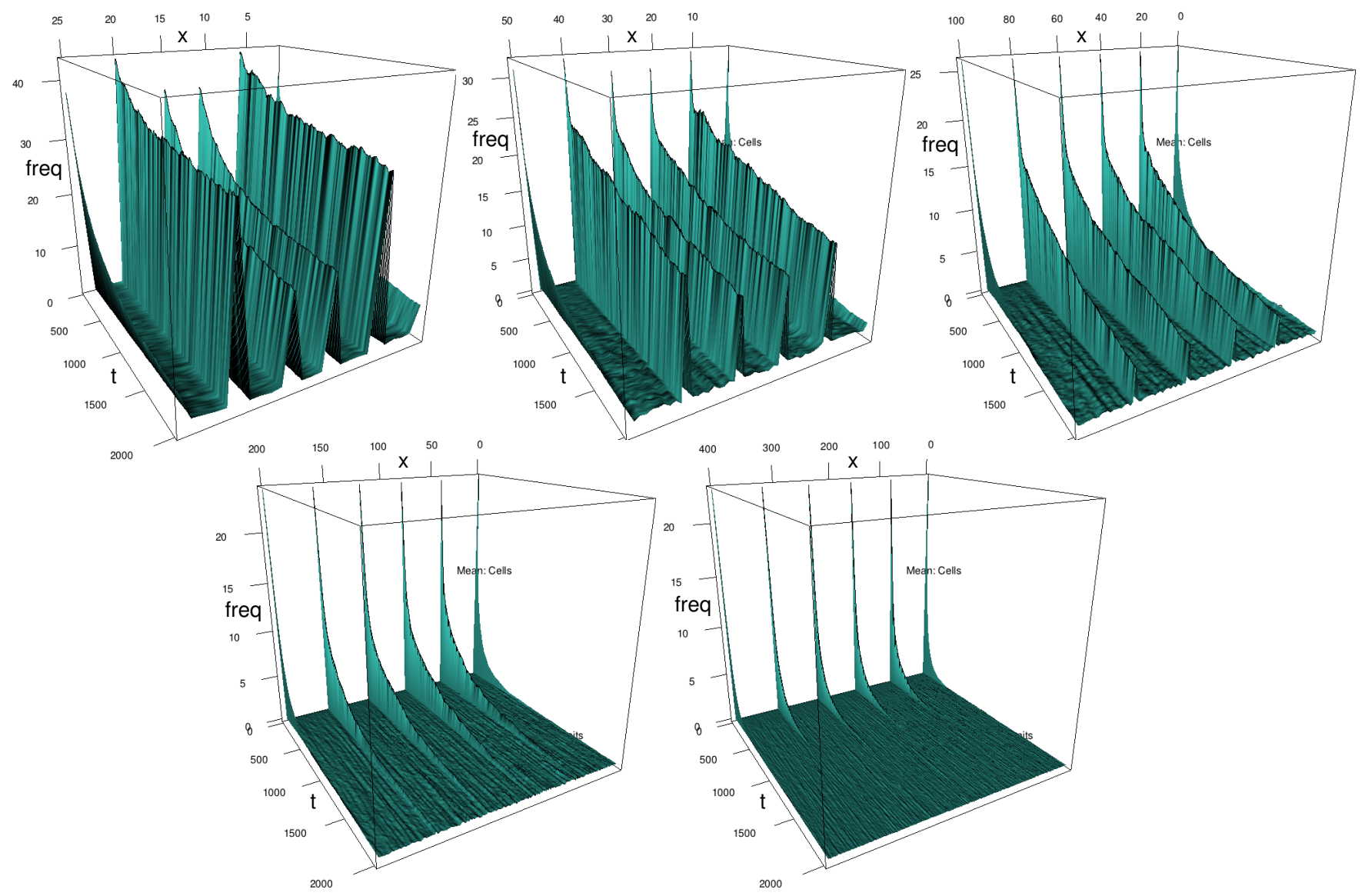

Figure 7: Stochastic reaction-diffusion system. Averages of 1000 runs for different grid densities. The numbers of grid nodes appear on the top of each panel.

\subsection{Deterministic system}

Figure 6 shows cross sections of time evolution of pre-cancerous cells density in the perturbed deterministic system for different number of nodes: 25, 50, 100, 200, and 400. Orange lines that look like horizontal straight lines are the cosinusoidal perturbations of the corresponding spatially homogeneous steady state.

The spatially homogeneous steady states are twice larger in magnitude if the number of nodes are halved, and the opposite holds true if the number of nodes are doubled. Values at 2000 time 
units (red lines) do not follow the same behavior. Spikes seem to decrease to what appears to be a limiting value, as the number of nodes increase. In all cases, spikes form in a single node.

The same behavior and conclusion can be drawn for bound growth factor. Results are not shown because they look exactly the same as a rescaled version of pre-cancerous cells.

Free growth factor values (not shown) double as number of nodes half, and vice versa. Increased number of nodes favor smoother results.

\subsection{Stochastic system}

Simulations of the main case were also performed for the same number of nodes presented in the previous subsection, using as initial conditions the end values of the corresponding perturbed deterministic systems rounded to the nearest integer.

Results for pre-cancerous cells are presented in Figure 7, which seems to lead to the conclusion that the stochastic system sustains better the level of averaged spikes for coarser grids, and the opposite for finer grids.

Results for free growth factor are omitted. Lower numbers of nodes convey a cross-sectional structure to the averaged values, which is increasingly lost as the number of nodes increase.

\section{Discussion}

We presented the stochastic extension of a one-dimensional reaction-diffusion model of early carcinogenesis that exhibits Turing instabilities in its deterministic formulation. We compared 250 cases of each system by modifying three parameters: 1) the number of peaks of the cosine perturbation of the spatially homogeneous steady state, which constituted the initial conditions, 2) the diffusion coefficient, and 3) the grid density. These parameters are only relevant in the spatial system.

In the model lacking spatial dimensions the transients in the deterministic case are different than in the stochastic case (right column of Figure 2, where blue line depicts deterministic runs and red lines depict averages \pm 3 standard errors of 1000 runs). This phenomenon has been noticed before in the context of well-mixed systems and it has been traced to the Jensen inequality [11].

The spatial system is an extension of the non-spatial one and, as a consequence, shows a similar effect: no stochastic system could maintain, when averaged over a large number of realizations, the initial levels of the spikes generated by the corresponding deterministic system, exhibiting in all cases a sudden drop in spike levels.

Diffusion and grid density play a role in the ability of the stochastic system to maintain, when averaged over a large number of realizations, the initial structure of spikes after the drop. Higher diffusion coefficients favor preservation of the initial spikes. Moreover, for $\gamma=1$ and $\gamma=10$, the stochastic system maintains spike levels indefinitely. Regarding grid density, when using a diffusion coefficient too low to maintain spike levels $(\gamma=100)$, coarser grids make the decay more gradual whereas denser grids make it more steep. 
Simple versions of the model with $\mu=0$ and constant positive $\kappa$, which lend themselves to a more detailed analysis were studied in ref. [6]. It was shown that in this case regular spatially inhomogeneous stationary solutions were unstable. Consequently, the pattern observed in numerical simulations might be a dynamical structure with the maxima growing to infinity with time. Moreover, it was determined in ref. [6] that there existed discontinuous inhomogeneous steady states, which were also unstable. Numerical simulations suggested emergence of distributional inhomogeneous steady states.

The results of our current research, as presented in this paper, add to this complexity. Four versions of the carcinogenesis model, resulting from taking or not taking into account the stochastic and spatial effects may exhibit very different dynamics. In particular, the mathematically intriguing behavior of the deterministic model with spatial effects is partly destroyed when stochastic effects are also added. What is the relevance of these results for the true biological system? Only refined experimental results may help answer this question.

Much of spatial modeling in biology has been accomplished using partial differential equations without reference to stochasticity. Almost without exceptions, this style of modeling hinges upon presumption that continuity provides the glue which allows using the machinery of calculus. In the systems considered in this paper, the continuity acts to produce asymptotically irregular (spiky) solutions. Stochasticity causes, in the mean, these spikes to gradually dissolve. At the single realization level, the stochastic system preserves enough spikeness to be a model of spontaneous formation of early cancer foci. However, they are now frequently reversible (Figure 1), while new ones are appearing. The qualitative picture is more complicated as a result.

\section{Acknowledgments}

The authors thank two anonymous referees for their helpful comments. Also, discussions with Dr. Anna Marciniak-Czochra are gratefully acknowledged. RB was supported by the NIGMS grant GM086885. MK was supported by the Polish NCN grant NN514411936.

\section{References}

[1] A. Marciniak-Czochra, M. Kimmel. Reaction-difusion model of early carcinogenesis: The effects of influx of mutated cells. Mathematical Modelling of Natural Phenomena, 3 (2008), No. 7, 90-114.

[2] A. Marciniak-Czochra, M. Kimmel. Dynamics of growth and signaling along linear and surface structures in very early tumors. Computational \& Mathematical Methods in Medicine, 7 (2006), No. 2/3, 189-213.

[3] A. Marciniak-Czochra, M. Kimmel. Modelling of early lung cancer progression: Influence of growth factor production and cooperation between partially transformed cells. Math. Mod. Meth. Appl. Sci., 17S (2007), 1693-1719. 
[4] A. Marciniak-Czochra, M. Kimmel. Reaction-diffusion approach to modeling of the spread of early tumors along linear or tubular structures. Journal of Theoretical Biology, 244 (2006), No. 3, 375-387.

[5] A. Marciniak-Czochra, M. Ptashnyk. Derivation of a macroscopic receptor-based model using homogenization techniques. SIAM J. Math. Anal., 40 (2008), No. 1, 215-237.

[6] A. Marciniak-Czochra, G. Karch, K. Suzuki. Unstable patterns in reaction-diffusion model of early carcinogenesis. arXiv:1104.3592v1, (2011).

[7] R. Bertolusso. Computational models of signaling processes in cells with applications: Influence of stochastic and spatial effects. PhD thesis (2011), Rice University, Houston, TX.

[8] R. Erban, S. J. Chapman, P. Maini. A practical guide to stochastic simulations of reactiondiffusion processes. ArXiv e-prints, (2007), April.

[9] S. A. Isaacson, C. S. Peskin. Incorporating diffusion in complex geometries into stochastic chemical kinetics simulations. SIAM J. Scientific Computing, 28 (2006), No. 1, 47-74.

[10] A. Slepoy, A. P. Thompson, S. J. Plimpton. A constant-time kinetic monte carlo algorithm for simulation of large biochemical reaction networks. J. Chem. Phys., 128 (2008), May, 205101.

[11] J. Paulsson, O. G. Berg, M. Ehrenberg. Stochastic focusing: fluctuation-enhanced sensitivity of intracellular regulation. Proc. Natl. Acad. Sci. U.S.A., 97 (2000), June, 7148-53. 